\title{
Immunome database for marsupials and monotremes
}

\author{
Emily SW Wong ${ }^{1}$, Anthony T Papenfuss ${ }^{2}$ and Katherine Belov ${ }^{1 *}$
}

\begin{abstract}
Background: To understand the evolutionary origins of our own immune system, we need to characterise the immune system of our distant relatives, the marsupials and monotremes. The recent sequencing of the genomes of two marsupials (opossum and tammar wallaby) and a monotreme (platypus) provides an opportunity to characterise the immune gene repertoires of these model organisms. This was required as many genes involved in immunity evolve rapidly and fail to be detected by automated gene annotation pipelines.

Description: We have developed a database of immune genes from the tammar wallaby, red-necked wallaby, northern brown bandicoot, brush-tail possum, opossum, echidna and platypus. The resource contains 2,235 newly identified sequences and 3,197 sequences which had been described previously. This comprehensive dataset was built from a variety of sources, including EST projects and expert-curated gene predictions generated through a variety of methods including chained-BLAST and sensitive HMMER searches. To facilitate systems-based research we have grouped sequences based on broad Gene Ontology categories as well as by specific functional immune groups. Sequences can be extracted by keyword, gene name, protein domain and organism name. Users can also search the database using BLAST.

Conclusion: The Immunome Database for Marsupials and Monotremes (IDMM) is a comprehensive database of all known marsupial and monotreme immune genes. It provides a single point of reference for genomic and transcriptomic datasets. Data from other marsupial and monotreme species will be added to the database as it become available. This resource will be utilized by marsupial and monotreme immunologists as well as researchers interested in the evolution of mammalian immunity.
\end{abstract}

\section{Background}

Recently, two marsupial genomes and one monotreme genome have been sequenced: the grey short-tailed opossum (Monodelphis domestica; $7 \times$ coverage) [1], the tammar wallaby (Macropus eugenii; $2 \times$ ) (in prep.), and the platypus (Ornithorhynchus anatinus; 6x) [2]. Marsupial and monotreme lineages branched off approximately 148 My and 166 My ago from the lineage leading to eutherian mammals [3]. They hold a unique evolutionarily position providing a link to the reptilian phase of our ancestry. Combined with their unusual biological traits, they are capable of providing important insights to our understanding of mammalian biology and evolution.

\footnotetext{
* Correspondence: kathy.belov@sydney.edu.au

${ }^{1}$ Faculty of Veterinary Sciences, University of Sydney, NSW 2006, Australia Full list of author information is available at the end of the article
}

Genome sequencing has generated huge amounts of genomic data. This has expedited the identification of genes in these species. Despite the availability of genome assemblies, only the most phylogenetically conserved immune genes have been identified using automated gene annotation pipelines. Genes involved in the immune response are subject to intense selective pressure due to the need to overcome pathogenic challenges. As a result, it is common for immune genes, particularly those with immunomodulatory roles, to show very low levels of sequence conservation between species $[4,5]$. This has lead to many key immune molecules being missed by the Ensembl [6] and NCBI's Gnomon http:// www.ncbi.nlm.nih.gov genome annotation platforms. Less than a third of all opossum immune genes that were annotated using specialized search strategies by Wong et al. 2006 [7], Belov et al. 2006 [8] and Belov et al. 2007 [9], were predicted by the Ensembl pipeline [6].
C Biomed Central

C 2011 Wong et al; licensee BioMed Central Ltd. This is an Open Access article distributed under the terms of the Creative Commons Attribution License (http://creativecommons.org/licenses/by/2.0), which permits unrestricted use, distribution, and reproduction in any medium, provided the original work is properly cited. 
Aside from high levels of sequence divergence, many immune gene families have also evolved through rapid successions of gene loss and gain, resulting in a lack of direct orthologs. Hence, these genes are difficult to characterize through local pairwise similarity search algorithms, such as BLAST [10], which use a single gene sequence to query a database.

To overcome the lack of annotated sequence information for immune genes, targeted, manually-curated strategies were applied $[7,9,11,12]$. Identification of the most highly divergent sequences required an intensive combination of strategies incorporating hidden Markov model searches, exploitation of conserved syntenic regions, sensitive local search algorithms and gene prediction integrating extrinsic information $[7,9,11,12]$. Less divergent genes missed by Ensembl could be identified and annotated using chained-BLAST searches [9].

Here, we present a database of curated marsupial and monotreme immune sequences. We have included novel predicted and expressed sequences as well as previously annotated genes [7,9,11-45]. Examples of gene groups represented in the database include chemokines, interleukins, Natural Killer (NK) receptors, Major Histocompatibility Complex (MHC) antigens, surface receptors, antimicrobial peptides. Annotations derived from a transcriptomic analysis on a primary lymphoid organ have also been included [46]. Many of these genes (e.g. 209 expressed tammar genes) have not been annotated by Ensembl and their sequences are not curated by other public databases. The database consists of a simple interface, and features several methods for users to query the sequences. On entry to the database, sequences were further annotated to provide searchable functional information. Availability of a comprehensive gene set assists large-scale projects such as transcriptomic analysis and microarray studies. Also, it facilitates the development of marsupial- and monotreme-specific reagents allowing for detailed analyses of metatherian and prototherian immune responses.

\section{Construction and content}

IDMM was implemented using the Python web framework Django (version 1.1) [47] with a SQLite3 (version 3.6.3) database [48]. Data can be easily updated by approved managers through a simple web interface. Once sequences are added, they are automatically matched to HGNC names and GO terms through a BLAST search. Amino acid sequences are additionally searched against the Conserved Domain Database (CDD) [49] to create protein domain annotations. Sequences are stored in FASTA format and are identified by their sequence header description which includes the gene name and species name.

\section{Database content and data source}

A total of 2,935 genes, 602 expressed (538 tammar wallaby, 24 opossum, 16 platypus, 11 echidna, 6 red-necked wallaby, 4 brushtail possum and 3 bandicoot) and 2,333 predicted (1,639 opossum, 694 platypus), are currently stored in the database. The database includes 1,985 published sequences. We have integrated data from various published resources, which include expressed and predicted genes from opossum (1,663) [7-9,18,34, $35,38,39,43-45,50]$, tammar (37) [21-26,33,35-37,45], brushtail possum (4) $[18,20,27-29,40]$ echidna (11) $[14,17,19,30,32]$, bandicoot (3) [15], red-necked wallaby (6) [42] and platypus (261) [11-14,16,18,19,31,41]. Manually annotated gene families include: major histocompatibility complex (MHC), leucocyte receptor complex (LRC), cytokine, defensin, cathelicidin, natural killer complex (NKC) and Fc receptor genes. Both opossum and platypus sequences were annotated using a curated list of human immune genes from the IRIS database [51]. For predicted genes, candidate gene regions were first identified using either BLAST [10] or HMMER hidden Markov model [52] searches. Following this, best hits were either concatenated into genes or used to predict a full gene model using a gene prediction program. 516 wallaby genes were annotated based on opossum genes identified in Wong et al. 2006 [7] and Belov et al. 2007 [9]. Of these, at least 217 were not annotated by Ensembl (version 58). Wallaby reads were derived from the pyrosequencing of wallaby thymus transcriptomes and annotated using the wallaby (v1.0) genome assembly [46]. For each annotated wallaby gene there were often multiple, overlapping reads; these were assembled and included in the database (1,786 wallaby reads in total). 449 platypus gene sequences were obtained by concatenation of the highest-scoring IRIS BLAST hits against the platypus genome assembly (v5.0) (Unpublished). Of these, 366 genes were not annotated by Ensembl (version 58).

\section{Sequence annotation}

All reads were defined by their species name, a gene symbol, the method of identification and sequence type (nucleotide or amino acid). To facilitate the retrieval of genes associated with specific immune roles, we categorized genes based on nine functional terms. These include the broad categories of humoral and cellular immunity and components of the innate (inflammation and complement system) and adaptive (antigen processing and presenting and phagocytosis) immune responses, as well as genes with regulatory functions such as chemokines and transcription factors. To provide additional sequence-based and functional information, all sequences were automatically annotated upon submission to the database. Automatic annotation was 
performed by searching the human SWISS-PROT [53] database at NCBI [54] with the submitted sequences using the network BLAST client (netblast) [10]. This resulted in the association of sequences with the official human gene names [55], GO ontology terms [56], and, for protein sequences, domain names. The accession of the best hit from each BLAST search was retrieved and matched to a list of pre-generated accession-specific tags if the E-value was less than 1e-3. These tags were linked to human gene names and gene ontology annotations using Entrez Gene data [57].

\section{Utility and Discussion}

\section{User interface}

Users can interrogate the database and retrieve gene sequences through a variety of simple query tools. The search interface spans three webpages. From the main page, users can query the database through keyword, organism name, human gene name, protein domain name and by the method through which sequences were obtained (Figure 1). A link exists to a GO term browser where terms can be examined in a tree structure that supports the natural relationships between GO terms (Figure 2). Finally, the BLAST program is implemented for users to search against sequences in the database.

\section{Search by curated gene symbols}

All gene names determined through annotation can be browsed. All sequences have been annotated with a

\begin{tabular}{|c|c|}
\hline \multicolumn{2}{|l|}{$\mathrm{GO} \mathbf{B r}$} \\
\hline GO:0000003 reproduction & 13 \\
\hline GO:0001906 cell killing & $\underline{3}$ \\
\hline GO:0002376 immune system process & 1262 \\
\hline GO:0008152 metabolic process & $\overline{1983}$ \\
\hline GO:0009987 cellular process & $\underline{2162}$ \\
\hline GO:0016032 viral reproduction & $\underline{0}$ \\
\hline$\overline{\mathrm{GO}: 0022414}$ reproductive process & $\underline{55}$ \\
\hline GO:0022610 biological adhesion & $\underline{0}$ \\
\hline GO:0032501 multicellular organismal process & $\underline{303}$ \\
\hline$\overline{\mathrm{GO}: 0032502}$ developmental process & $\overline{388}$ \\
\hline GO:0034600 macromolecular complex organization & $\underline{164}$ \\
\hline GO:0040007 growth & 1 \\
\hline GO:0040011 locomotion & $\underline{0}$ \\
\hline GO:0043473 pigmentation & $\underline{2}$ \\
\hline \multicolumn{2}{|c|}{ GO:0043933 macromolecular complex subunit organization $\underline{0}$} \\
\hline GO:0048511 rhythmic process & 10 \\
\hline GO:0050896 response to stimulus & 879 \\
\hline GO:0051179 localization & 4 \\
\hline GO:0051234 establishment of localization & $\underline{723}$ \\
\hline GO:0051704 multi-organism process & $\underline{246}$ \\
\hline GO:0065007 biological regulation & 1652 \\
\hline GO:0048518 positive regulation of biological process & $\underline{5}$ \\
\hline GO:0048519 negative regulation of biological process & $\underline{24}$ \\
\hline GO:0050789 regulation of biological process & $\underline{1573}$ \\
\hline
\end{tabular}

gene name based on the human gene symbol, with the exception of lineage-specific expansions, such as NKC genes and MHC genes. Characterized species-specific expansions (i.e. without human one-to-one orthologs)

Search by annotated gene names

Search by functional category

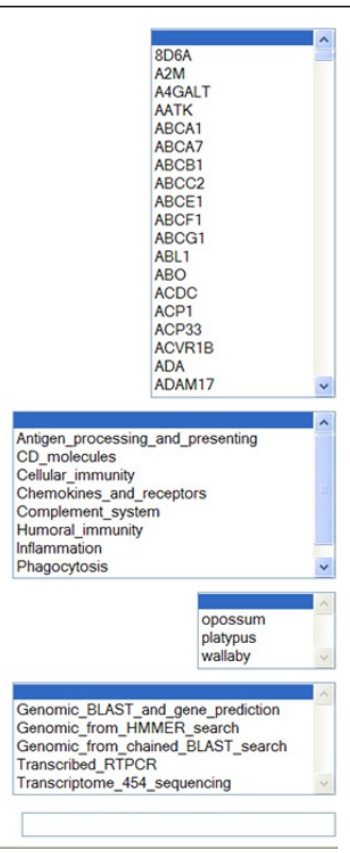

Figure 1 Main search page of IDMM. 
are labelled using the gene family name followed by a unique set of numeric identifiers.

\section{Keyword search}

A simple keyword search permits users to query the database using any string of characters from any description line in FASTA sequences, human gene descriptions and GO names. All FASTA descriptions contain the common name of the species from which the sequences were derived. In addition to terms present in the FASTA header description, users may also search terms generated by automatic annotation which include full gene name (in addition to HGNC symbol) and GO terms. Only sequences of high similarity (E-value $<1 \mathrm{e}-$ 3) to human genes were automatically annotated. Two keyword searches are available: one for exact but caseinsensitive match in sequence headers only and one which matches all terms containing the keyword from all associations, including, for example, GO descriptions.

\section{Search by sequence identification method}

Sequence retrieval via the initial sequence identification method (e.g. BLAST) allows simple discrimination between expressed and predicted genes. It is important to note that while chained high scoring BLAST alignments may provide more sequence information, the predicted sequence may not be identical to the actual transcribed sequence. We have also provided information on the identification method used on each sequence label.

\section{Search by HGNC gene symbols}

To facilitate the retrieval of marsupial and monotreme homologs to human genes, a list of human gene symbols is available for browsing. We queried marsupial and monotreme database sequences against all human proteins and linked the best hits based on the E-value. The resultant annotations are, in effect, reciprocal best hits of predicted genes. By comparison of gene symbols, users can rapidly determine the accuracy of an ortholog assignment. This strategy provides a measure of the level of confidence in the assigned gene name.

\section{Search by conserved protein domains}

To facilitate rapid identification of gene family members, users can search for sequences based on annotated protein functional units from the Conserved Domain Database (CDD). CDD names can be browsed by list and by hyperlinks via 'tag cloud'. Conserved domain annotations are only available for amino acid sequences.

\section{Search based on GO terms}

Users may interrogate biological and molecular functional processes and structural components through a
GO browser (Figure 2). The browser follows the tree-like hierarchy of GO data by linking general terms to specific terms. The GO terms associated with monotreme and marsupial sequences are inferred through sequence similarity to human Entrez gene annotations. For each term the number of associated database sequences is located after the GO name. By clicking on this number users can extract all associated sequences. Note that Entrez GO terms often miss higher level terms which will underestimate the number of genes in a category. Therefore, it is advisable to browse through GO child terms.

\section{Search through the BLAST interface}

Users can direct BLAST queries against the sequence database. Users can perform nucleotide, translated nucleotide and protein searches. Results are presented in standard BLAST text output format.

\section{Sequence retrieval}

With the exception of BLAST searches, sequences are viewed through a standard retrieval interface. FASTA headers uniquely identify each sequence. In addition to the option of retrieving reads individually, users may choose to retrieve all identified sequences at once. Users can also fetch all associated annotations for each sequence. An option to display amino acid or nucleotide sequences is available.

\section{Conclusion}

Targeted search strategies for immune genes and gene families have led to the annotation of previously unidentified marsupials and monotreme genes in the recent genome assemblies of the opossum, tammar wallaby and platypus. Genes involved in immunity are generally poorly annotated in genome assemblies due to their high rate of sequence divergence and gene duplications. This high sequence divergence of marsupial and monotreme immune genes also renders them difficult to isolate with classical lab techniques. IDMM provides easy access to marsupial and monotreme immune sequences. It hosts a catalogue of novel and integrated sets of published genes, searchable through a simple-to-use and fast interface. The availably of these sequences will facilitate the development of species-specific immunological reagents, enabling accurate studies of immune responses in these species. This database will be useful for comparative studies of immunity.

\section{Availability and requirements}

IDMM is publicly available at http://hp580.angis.org.au/ tagbase/gutentag/.

\section{Acknowledgements}

We thank Dao Mai, Lee Render, Dr Matthew Hobbs and Mette Lille for technical advice and suggestions for improving the database. EW was 
supported by an ARC Kangaroo Genomics and Jean Walker postgraduate scholarship.

\section{Author details}

${ }^{1}$ Faculty of Veterinary Sciences, University of Sydney, NSW 2006, Australia. ${ }^{2}$ Bioinformatics Division, The Walter and Eliza Hall Institute for Medical Research, Parkville, Victoria 3050, Australia.

\section{Authors' contributions}

EW sourced and identified the sequences, designed and implemented the database and web interface. AP, KB and EW conceived the concept. EW wrote the manuscript and $\mathrm{KB}$ and $\mathrm{AP}$ edited the manuscript. All authors read and approved the final manuscript.

Received: 7 April 2010 Accepted: 19 August 2011

Published: 19 August 2011

\section{References}

1. Mikkelsen TS, Wakefield MJ, Aken B, Amemiya CT, Chang JL, Duke S, Garber M, Gentles AJ, Goodstadt L, Heger A, et al: Genome of the marsupial Monodelphis domestica reveals innovation in non-coding sequences. Nature 2007, 447(7141):167-177.

2. Warren WC, Hillier LW, Marshall Graves JA, Birney E, Ponting CP, Grutzner F, Belov K, Miller W, Clarke L, Chinwalla AT, et al: Genome analysis of the platypus reveals unique signatures of evolution. Nature 2008, 453(7192):175-183.

3. Bininda-Emonds OR, Cardillo M, Jones KE, MacPhee RD, Beck RM, Grenyer R, Price SA, Vos RA, Gittleman JL, Purvis A: The delayed rise of present-day mammals. Nature 2007, 446(7135):507-512.

4. Zelus D, Robinson-Rechavi M, Delacre M, Auriault C, Laudet V: Fast evolution of interleukin-2 in mammals and positive selection in ruminants. Journal of Molecular Evolution 2000, 51(3):234-244.

5. Kaiser P, Poh TY, Rothwell L, Avery S, Balu S, Pathania US, Hughes S, Goodchild M, Morrell S, Watson M, et al: A genomic analysis of chicken cytokines and chemokines. Journal of Interferon \& Cytokine Research: The Official Journal of the International Society for Interferon and Cytokine Research 2005, 25(8):467-484.

6. Curwen V, Eyras E, Andrews TD, Clarke L, Mongin E, Searle SMJ, Clamp M: The Ensembl automatic gene annotation system. Genome Research 2004 14(5):942-950.

7. Wong E, Young L, Papenfuss A, Belov K: In silico identification of opossum cytokine genes suggests the complexity of the marsupial immune system rivals that of eutherian mammals. Immunome Research 2006 2(1):4-4

8. Belov K, Deakin JE, Papenfuss AT, Baker ML, Melman SD, Siddle HV, Gouin N, Goode DL, Sargeant TJ, Robinson MD, et al: Reconstructing an Ancestral Mammalian Immune Supercomplex from a Marsupial Major Histocompatibility Complex. PLoS Biology 2006, 4(3):e46 EP, -e46 EP -

9. Belov K, Sanderson CE, Deakin JE, Wong ES, Assange D, McColl KA, Gout A, de Bono B, Barrow AD, Speed TP, et al: Characterization of the opossum immune genome provides insights into the evolution of the mammalian immune system. Genome Res 2007, 17(7):982-991.

10. Altschul SF, Madden TL, Schaffer AA, Zhang J, Zhang Z, Miller W, Lipman DJ: Gapped BLAST and PSI-BLAST: a new generation of protein database search programs. Nucleic Acids Res 1997, 25(17):3389-3402.

11. Wong ESW, Papenfuss AT, Miller RD, Belov K: Hatching time for monotreme immunology. Australian Journal of Zoology 2009 57(4):185-198

12. Wong ES, Sanderson CE, Deakin JE, Whittington CM, Papenfuss AT, Belov K: Identification of natural killer cell receptor clusters in the platypus genome reveals an expansion of C-type lectin genes. Immunogenetics 2009, 61(8):565-579.

13. Whittington CM, Papenfuss AT, Bansal P, Torres AM, Wong ES, Deakin JE, Graves T, Alsop A, Schatzkamer K, Kremitzki C, et al: Defensins and the convergent evolution of platypus and reptile venom genes. Genome Res 2008, 18(6):986-994.

14. Belov K, Lam MKP, Hellman L, Colgan DJ: Evolution of the major histocompatibility complex: Isolation of class II beta cDNAs from two monotremes, the platypus and the short-beaked echidna. Immunogenetics 2003, 55(6):402-411.
15. Baker ML, Miller RD: Evolution of mammalian CD1: marsupial CD1 is not orthologous to the eutherian isoforms and is a pseudogene in the opossum Monodelphis domestica. Immunology 2007, 121(1):113-121.

16. Johansson J, Salazar JN, Aveskogh M, Munday B, Miller RD, Hellman L: High variability in complementarity-determining regions compensates for a low number of $\mathrm{V}$ gene families in the lambda light chain locus of the platypus. European Journal of Immunology 2005, 35(10):3008-3019.

17. Nowak MA, Parra ZE, Hellman L, Miller RD: The complexity of expressed kappa light chains in egg-laying mammals. Immunogenetics 2004 56(8):555-563

18. Miska KB, Hellman L, Miller RD: Characterization of beta(2)-microglobulin coding sequence from three non-placental mammals: the duckbill platypus, the short-beaked echidna, and the grey short-tailed opossum. Developmental and Comparative Immunology 2003, 27(3):247-256.

19. Miska KB, Harrison GA, Hellman L, Miller RD: The major histocompatibility complex in monotremes: an analysis of the evolution of Mhc class I genes across all three mammalian subclasses. Immunogenetics 2002, 54(6):381-393.

20. Belov K, Harrison GA, Miller RD, Cooper DW: Molecular cloning of four lambda light chain cDNAs from the Australian brushtail possum Trichosurus vulpecula. European Journal of Immunogenetics: Official Journal of the British Society for Histocompatibility and Immunogenetics 2002, 29(2):95-99.

21. Old JM, Deane EM, Harrison GA: Molecular characterisation of the tammar wallaby (Macropus eugenii) CD3 epsilon chain cDNA. Molecular Immunology 2001, 38(5):359-364.

22. Daly KA, Digby MR, Lefévre C, Nicholas KR, Deane EM, Williamson P: Identification, characterization and expression of cathelicidin in the pouch young of tammar wallaby (Macropus eugenii). Comparative Biochemistry and Physiology Part B, Biochemistry \& Molecular Biology 2008 149(3):524-533.

23. Harrison GA, McNicol KA, Deane EM: Interferon alpha/beta genes from a marsupial, Macropus eugenii. Developmental and Comparative Immunology 2004, 28(9):927-940.

24. Harrison GA, Deane EM: cDNA sequence of the lymphotoxin beta chain from a marsupial, Macropus eugenii (Tammar wallaby). Journal of Interferon \& Cytokine Research: The Official Journal of the International Society for Interferon and Cytokine Research 1999, 19(10):1099-1102.

25. Harrison GA, Broughton MJ, Young LJ, Cooper DW, Deane EM: Conservation of $3^{\prime}$ untranslated region elements in tammar wallaby (Macropus eugenii) TNF-alpha mRNA. Immunogenetics 1999, 49(5):464-467.

26. Harrison GA, Deane EM: cDNA cloning of lymphotoxin alpha (LT-alpha) from a marsupial, Macropus eugenii. DNA Sequence: The Journal of DNA Sequencing and Mapping 2000, 10(6):399-403.

27. Wedlock DN, Goh LP, Parlane NA, Buddle BM: Molecular cloning and physiological effects of brushtail possum interleukin-1beta. Veterinary Immunology and Immunopathology 1999, 67(4):359-372.

28. Wedlock DN, Aldwell FE, Buddle BM: Molecular cloning and characterization of tumor necrosis factor alpha (TNF-alpha) from the Australian common brushtail possum, Trichosurus vulpecula. Immunology and Cell Biology 1996, 74(2):151-158

29. Cui S, Selwood L: cDNA cloning, characterization, expression and recombinant protein production of leukemia inhibitory factor (LIF) from the marsupial, the brushtail possum (Trichosurus vulpecula). Gene 2000, 243(1-2):167-178

30. Vernersson $M$, Aveskogh $M$, Hellman L: Cloning of IgE from the echidna (Tachyglossus aculeatus) and a comparative analysis of epsilon chains from all three extant mammalian lineages. Developmental and Comparative Immunology 2004, 28(1):61-75.

31. Parra ZE, Arnold T, Nowak MA, Hellman L, Miller RD: TCR gamma chain diversity in the spleen of the duckbill platypus (Ornithorhynchus anatinus). Developmental and Comparative Immunology 2006, 30(8):699-710.

32. Belov K, Miller RD, Ilijeski A, Hellman L, Harrison GA: Isolation of monotreme T-cell receptor alpha and beta chains. Immunogenetics 2004 56(3):164-169.

33. Harrison GA, Taylor CL, Miller RD, Deane EM: Primary structure and variation of the T-cell receptor delta-chain from a marsupial, Macropus eugenii. Immunology Letters 2003, 88(2):117-125.

34. Parra ZE, Baker ML, Schwarz RS, Deakin JE, Lindblad-Toh K, Miller RD: A unique $T$ cell receptor discovered in marsupials. Proceedings of the 
National Academy of Sciences of the United States of America 2007, 104(23):9776-9781.

35. Duncan LG, Nair SV, Deane EM: The marsupial CD8 gene locus: molecular cloning and expression analysis of the alpha and beta sequences in the gray short-tailed opossum (Monodelphis domestica) and the tammar wallaby (Macropus eugenii). Veterinary Immunology and Immunopathology 2009, 129(1-2):14-27.

36. Siddle HV, Deakin JE, Baker ML, Miller RD, Belov K: Isolation of major histocompatibility complex Class I genes from the tammar wallaby (Macropus eugenii). Immunogenetics 2006, 58(5-6):487-493.

37. Daly KA, Digby M, Lefèvre $C$, Mailer $S$, Thomson P, Nicholas K, Williamson P: Analysis of the expression of immunoglobulins throughout lactation suggests two periods of immune transfer in the tammar wallaby (Macropus eugenii). Veterinary Immunology and Immunopathology 2007, 120(3-4):187-200.

38. Parra ZE, Baker ML, Lopez AM, Trujillo J, Volpe JM, Miller RD: TCR mu recombination and transcription relative to the conventional TCR during postnatal development in opossums. Journal of Immunology (Baltimore, Md: 1950) 2009, 182(1):154-163.

39. Baker ML, Melman SD, Huntley J, Miller RD: Evolution of the opossum major histocompatibility complex: evidence for diverse alternative splice patterns and low polymorphism among class I genes. Immunology 2009, 128(1 Suppl):e418-431-e418-431.

40. Adamski FM, Demmer J: Immunological protection of the vulnerable marsupial pouch young: two periods of immune transfer during lactation in Trichosurus vulpecula (brushtail possum). Developmental and Comparative Immunology 2000, 24(5):491-502.

41. Vernersson M, Belov K, Aveskogh M, Hellman L: Cloning and structural analysis of two highly divergent $\lg A$ isotypes, $\lg A 1$ and $\lg A 2$ from the duck billed platypus, Ornithorhynchus anatinus. Molecular Immunology 2010, 47(4):785-791.

42. Schneider S, Vincek V, Tichy H, Figueroa F, Klein J: MHC class II genes of a marsupial, the red-necked wallaby (Macropus rufogriseus): identification of new gene families. Molecular Biology and Evolution 1991, 8(6):753-766.

43. Miller RD, Grabe $H$, Rosenberg $G H: V(H)$ repertoire of a marsupial (Monodelphis domestica). Journal of Immunology (Baltimore, Md: 1950) 1998, 160(1):259-265.

44. Lucero JE, Rosenberg GH, Miller RD: Marsupial light chains: complexity and conservation of lambda in the opossum Monodelphis domestica. Journal of Immunology (Baltimore, Md: 1950) 1998, 161(12):6724-6732.

45. Duncan LG, Nair SV, Deane EM: Molecular characterisation and expression of CD4 in two distantly related marsupials: the gray short-tailed opossum (Monodelphis domestica) and tammar wallaby (Macropus eugenii). Molecular Immunology 2007, 44(15):3641-3652.

46. Wong ES, Papenfuss AT, Heger A, Hsu AL, Ponting CP, Miller R, Renfree MB, Gibbs RA, Belov K: Transcriptomic analysis supports similar functional roles for the two thymuses of the tammar wallaby. BMC Genomics.

47. Django. [http://www.djangoproject.com/].

48. SQLite. [http://sqlite.org/].

49. Marchler-Bauer A, Panchenko AR, Shoemaker BA, Thiessen PA, Geer LY, Bryant SH: CDD: a database of conserved domain alignments with links to domain three-dimensional structure. Nucleic Acids Res 2002, 30(1):281-283.

50. Miska KB, Miller RD: Marsupial Mhc class I: classical sequences from the opossum, Monodelphis domestica. Immunogenetics 1999, 50(1-2):89-93.

51. Kelley J, de Bono B, Trowsdale J: IRIS: a database surveying known human immune system genes. Genomics 2005, 85(4):503-511.

52. Eddy SR: Profile hidden Markov models. Bioinformatics 1998, 14(9):755-763.

53. Boeckmann B, Bairoch A, Apweiler R, Blatter MC, Estreicher A, Gasteiger E, Martin MJ, Michoud K, O'Donovan C, Phan I, et al: The SWISS-PROT protein knowledgebase and its supplement TrEMBL in 2003. Nucleic Acids Res 2003, 31(1):365-370.

54. National Center for Biotechnology Information (NCBI). [http://www.ncbi. nIm.nih.gov/].

55. HUGO Gene Nomenclature Committee. [http://www.genenames.org/]

56. Ashburner M, Ball CA, Blake JA, Botstein D, Butler H, Cherry JM, Davis AP, Dolinski K, Dwight SS, Eppig JT, et al: Gene ontology: tool for the unification of biology. The Gene Ontology Consortium. Nat Genet 2000, 25(1):25-29.

57. Maglott D, Ostell J, Pruitt KD, Tatusova T: Entrez Gene: gene-centered information at NCBI. Nucleic Acids Res 2007, , 35 Database: D26-31. doi:10.1186/1471-2172-12-48

Cite this article as: Wong et al: Immunome database for marsupials and monotremes. BMC Immunology 2011 12:48.

\section{Submit your next manuscript to BioMed Central and take full advantage of:}

- Convenient online submission

- Thorough peer review

- No space constraints or color figure charges

- Immediate publication on acceptance

- Inclusion in PubMed, CAS, Scopus and Google Scholar

- Research which is freely available for redistribution 